\title{
Integrating Internet of Things (IoT) in the Measurements and Instrumenta- tion Course
}

\section{Dr. Mohammad Habibi P.E., University of Wisconsin, Platteville}

Mohammad Habibi is an Assistant Professor in the Department of Electrical and Computer Engineering at the University of Wisconsin-Platteville. Prior to coming to the UW-Platteville, he was an assistant professor of Integrated Engineering at Minnesota State University-Mankato. He earned his Ph.D. degree in Electrical Engineering from the University of Wisconsin-Milwaukee in 2010. His primary research interests are in the field of signal processing, dielectric spectroscopy, and sensors. Specifically, he is interested in developing novel medical devices. In addition to his technical research, he is also an active member of the American Society of Engineering Education (ASEE) and conducts research in engineering education. 
Integrating Internet of Things (IoT) in the Measurements and Instrumentation Course 


\section{Introduction}

The Internet of Things (IoT) is defined as a network of sensors/actuators, where these devices collect and exchange data via the internet [1]. The rapid growth of the IoT is revolutionizing our world and the significant drop in price for typical IoT components is allowing us to innovate new devices. The IoT is developed to integrate physical world into the computer-based systems by allowing physical variables to be sensed or controlled remotely via existing networks. This results in improved efficiency, accuracy, and provided economic benefit, in addition to reducing human intervention. The true value of the Internet of Things is based on the data collected from connected devices, which enable us to run analytics, optimizing our technology and consequently our life.

Currently, most schools have developed or are in the process of integrating the IoT in their curricula. For instance, the Department of Computer Engineering at Santa Clara University has developed and offered a graduate-level course in the Internet of Things [2]. Their course is composed of a series of lectures, and it includes a laboratory component. In the University of Minnesota, an IoT lab module has been developed and added to an electrical engineering freshman-level $\mathrm{C}++$ programming course [3]. Their $\mathrm{C}++$ programming course consists of 15weeks, two one hour and half lectures and one three-hour lab session per week. Half of their labs are IoT related, consisting of device setup, input/output devices, cloud connectivity, and simple multitasking. The challenges in offering the IoT course has been identified as the content of the course and at what level the course needs to be introduced [4]. The IoT includes both hardware and software components, covering extensive material. It has been noted that it is a challenge to have a unified curriculum for the IoT [5].

Measurements and Instrumentation course is offered in many engineering and technology schools to introduce undergraduate engineering students to the measurement principles and instruments used for measuring physical quantities. In recent years, advanced topics such as smart sensors, intelligent instruments, digital components (e.g. storage, displays, interfaces, etc.) have been added to the content of the course. Applications of this course include, but are not limited to, building automation, industrial control systems, and safety controls. However, the new concept of the IoT is not commonly covered by this course.

At the University of Wisconsin-Platteville, a new IoT module has been developed and added to the existing measurements and instrumentation course. The module consists of five hours of lectures, which covers the theory, and a design lab project. The lecture covers the IoT concepts such as analytics, webservers, communication and data protocols. The design lab project requires students to implement the theory into a practical application.

In this paper, the detail of the module is described. It is expected that our experience can be helpful to other engineering instructors seeking to develop an IoT course or just adding a section to their measurements and instrumentation course. 


\section{Measurements and Instrumentation}

Measurement and Instrumentation (M\&I) course is commonly offered for electrical and mechanical engineering or technology students. The main objective of the course is to introduce undergraduate engineering students to the fundamentals of measuring physical quantities and designing instruments for performing measurements. The topics taught in this course, include error analysis, signal conditioning circuits, sensor applications \& technology, and noise analysis. The course has numerous applications such as industrial automation, building controls, public safety, and consumer electronics. In the University of Wisconsin-Platteville, the M\&I course was developed in 2017 and has been taught every spring since then. The course compromises of three hours of lectures and two hours of lab, weekly. For the hands-on portion of the course, the students are required to complete three mini and one final projects. The final project has to be a multi-sensors/actuators product with the IoT capabilities. In the final project, the students enrolled in the course, work in a team setting with three to five members. There are multiple textbooks are available for this course; but we have used a number of white papers and two textbooks [6,7]. The content and learning objectives of the Measurements and Instrumentation course are indicated in Table 1. As one of the course objectives, the students must be able to understand the fundamental of IoT, in addition to design and implement IoT systems.

Table 1. The content and learning objectives of Measurements and Instrumentation course offered at the University of WisconsinPlatteville

\begin{tabular}{|c|c|}
\hline Content & Learning objectives \\
\hline $\begin{array}{ll}> & \text { Measurement systems and } \\
& \text { instrument characteristics. } \\
> & \text { Error analysis. } \\
> & \text { Noise and interference in } \\
& \text { instrumentation. } \\
> & \text { Signal conditioning. } \\
> & \text { Internet of Things (IoT). } \\
> & \text { Sensor applications. } \\
> & \text { Data acquisition, digital } \\
& \text { interfaces (A/D and D/A). } \\
> & \text { Discussion of specific sensor } \\
& \text { systems. }\end{array}$ & $\begin{array}{l}>\text { Understand the fundamental principles of } \\
\text { measurement and uncertainty. } \\
>\text { Ability to analyze measurement systems. } \\
>\text { Ability to design, calibrate, and characterize a } \\
\text { measurement system to measure mechanical or } \\
\text { electrical variables. } \\
>\quad \text { Understand the fundamental of IoT and be able to } \\
\text { design and implement an IoT system } \\
>\quad \text { Gain an understanding of some of the specific sensor } \\
\text { systems. } \\
>\text { Ability to use Lab VIEW in implementing a modern } \\
>\text { measurement system. } \\
>\text { Understand modern sensor systems for measuring a } \\
\text { variety of physical quantities. } \\
>\text { Ability to work in a multidisciplinary team. } \\
>\text { Ability to communicate effectively. }\end{array}$ \\
\hline
\end{tabular}




\section{Course Assessments}

The detail of the course assessment is shown in the Table 2. The course assessment consists of exams, projects, quizzes, and a survey paper. There are eleven weekly quizzes, only ten in which are counted toward the final grade. Usually, one or two quizzes are pulled from the IoT lectures. The quizzes are multiple choice and each quiz is comprised of multiple questions. The students take these online quizzes over the weekend. A sample quiz is shown in the Table 3. The survey paper entails a description of a new instrument, however many students preferred IoT-related topics.

Table 2. M\&I course assessments

\begin{tabular}{|l|l|l|}
\hline Course components & Percentage & IoT module \\
\hline Exams (Exam I ,II ,III) & $50 \%$ & \\
\hline Projects (4 Projects) & $30 \%$ & One of the project \\
\hline Quiz (10 weekly quiz) & $10 \%$ & One or two quizzes \\
\hline Survey paper & $10 \%$ & Students' preferences \\
\hline
\end{tabular}

Table 3. A sample quiz related to IoT topics discussed in the lecture

\begin{tabular}{|l|l|}
\hline Quiz \# 8 & Multiple-Choice \\
\hline Which one the following communication protocol & a) ZigBee \\
does offer the highest data rate? & b) Bluetooth \\
& c) WiFi \\
& d) DASH7 \\
\hline Which one the following communication protocol & a) ZigBee \\
does have the highest power usage? & b) Bluetooth \\
& c) WiFi \\
d) GSM/3G \\
\hline Which one the following communication protocol & a) ZigBee \\
does have the highest coverage? & b) Bluetooth \\
& c) WiFi \\
& d) LoRaWAN \\
\hline Which one the following communication protocol & a) ZigBee \\
does have the lowest latency? & b) Bluetooth \\
& c) WiFi \\
& d) DSRC/WAVE \\
\hline
\end{tabular}

\section{Projects}

The M\&I is an intensive design-based and hands-on course. The students are required to bridge lecture material into the hands-on projects. There are four design projects in this course; with the exception of project 1 , all other three projects are related. In project 1 , the students design and prototype a temperature measuring device using a Resistive Temperature Dependent (RTD). In this project, the students design a signal conditioning circuit using two methods: Wheatstone bridge, and an Anderson current loop. After completing project 1, a menu of the final projects is published, containing projects proposed by the students, faculty and local industry. Each project consists of multiple sensors/actuators. The students are given an opportunity to choose their projects and subsequently, their sensors. In project 2, the students use the knowledge of project 1 
and design a signal conditioning circuit for their sensors. Depending on the final product, their sensors can be pressure, force, light, humidity, conductivity, etc. To complete the project 2 , the students are required to calibrate and test their devices. The project 3 is to add the IoT capabilities such as remote sensing/controlling to the project 2 . Table 4 summarizes the projects and objectives.

Table 4. Course projects and their objectives

\begin{tabular}{|l|l|l|}
\hline 1 & Project & Objective \\
\hline 2 & Tranperature measurement & $\begin{array}{l}\text { Design, build, and test a temperature -measuring device using an RT D and OpAmps. } \\
\text { Understand the fundamental of signal conditioning. Design three different signal- } \\
\text { conditioning circuits: Voltage divider, Anderson current loop and a Wheatstone bridge. }\end{array}$ \\
\hline 3 & IoT & $\begin{array}{l}\text { Design, fabrication and testing of a transducer. Learn how to design GUI (Graphical } \\
\text { User Interface) using LabVIEW, Learn how to design a control circuit. }\end{array}$ \\
\hline 4 & Final project: team-based & $\begin{array}{l}\text { Learn how to setup your first Internet of Things project. } \\
\text { Learn Node-Red, MQTT, ESP8266, AWS, and Analytics }\end{array}$ \\
\hline
\end{tabular}

Regarding the final project, the students integrate their designs into one product. The final project is completed in a team-based setting. Figure 1 illustrates a block diagram of an instrument with multiple sensing/controlling and the IoT capabilities. A list of projects completed in the spring of 2018 is shown in the Table 5.

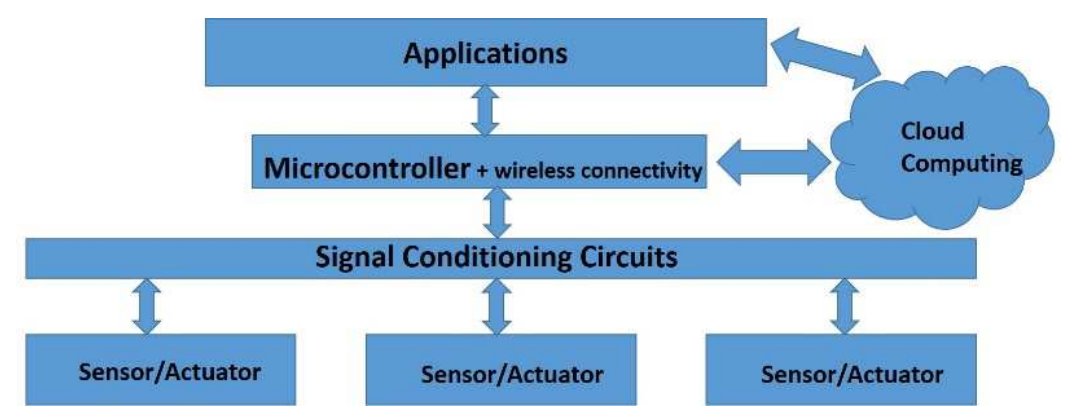

Figure 1. Instrument with IoT capabilities

\section{IoT Module}

The IoT can be an independent course to cover both hardware and software, or can be a module. We implemented an IoT module compromising of five hour of lectures (four sessions of one hour and fifteen minutes) and a lab project into the M\&I course. Although five hours are not enough to cover all the IoT concepts in depth, it is still enough for the electrical engineering students to have a short breath of the topics. In this module, the students learned concepts such as applications, challenges, network protocols, and smart connected objects. The content covered in the module is shown in Table 6. In addition to the lectures, students were required to design and implement an IoT device (project 3 as described above). 


\begin{tabular}{|c|c|}
\hline Project & Description \\
\hline $\begin{array}{l}\text { Smart } \\
\text { Blender }\end{array}$ & $\begin{array}{l}\text { Developing a way to automatically turn off a food blender or processor once the smoothie comes to a certain } \\
\text { consistency, and to adjust settings via cellphone. When the perfect smoothie is ready, the blender shuts off. This } \\
\text { prevents over-blending. This costs little to manufacture, saves time in the kitchen and is easy to use. The product } \\
\text { was made of two sensors: a Hall effect current sensor and a load sensor with a signal conditioning circuit to amplify } \\
\text { the signal from the load cell. By monitoring both the current through the blender motor and weight of the contents of } \\
\text { the blender, the condition of content can be predicted. A graphical interface, android app, was designed so a user can } \\
\text { adjust the setting or remotely control the blender. }\end{array}$ \\
\hline $\begin{array}{l}\text { Power line } \\
\text { monitoring }\end{array}$ & $\begin{array}{l}\text { A power line monitoring system was designed to monitor current, temperature, inclination and tension of power } \\
\text { lines. The system consists of four sensors: current, temperature, inclination, and tension. Four sensors were chosen } \\
\text { and signals were conditioned to output } 0 \mathrm{~V} \text { when the line is in normal condition and } 1 \mathrm{~V} \text { when fault happens. Each } \\
\text { sensor works simultaneously with one another. The analog reading from the circuits is read by the ESP } 8266 \text { chip, } \\
\text { which then transfers the data to the web server and from the web server the data is published to the GUI, where the } \\
\text { data can be read by the user. }\end{array}$ \\
\hline $\begin{array}{l}\text { Smart } \\
\text { Parking } \\
\text { Management }\end{array}$ & $\begin{array}{l}\text { The purpose of this project was to determine the vacancy of parking spaces in any parking lot using advanced image } \\
\text { processing techniques. These techniques were implemented using Raspberry Pi } 3 \text { with the Raspberry Pi Camera } \\
\text { Module V2. The accuracy for this project should be a minimum of } 80 \text { percent on a sunny day from 8:00 am to 4:00 } \\
\text { pm. We used the parking lot behind Engineering Hall, which contains } 83 \text { spots. A server backend and a graphical } \\
\text { user interface were implemented by software engineers and served via web application. }\end{array}$ \\
\hline $\begin{array}{l}\text { Smart } \\
\text { Hydroponic } \\
\text { systems }\end{array}$ & $\begin{array}{l}\text { Hydroponic systems traditionally require an extreme amount of maintenance to produce a successful yield. The } \\
\text { objective of this project was to automate the process of hydroponics. We implemented } 5 \text { sensors to monitor and } \\
\text { automate the process of hydroponics. The parameters our system tracks were soil moisture, light intensity, electrical } \\
\text { conductivity of nutrient the solution (to determine level of nutrients), temperature of the nutrient solution, and } \\
\text { temperature of the air. A system prototype has been designed which implements the five sensors listed above in } \\
\text { conjunction with a cloud hosted data server, designed by a team of software engineers, which allows the user to } \\
\text { interface with the sensor data in real time. The prototype provided remote access to real time sensor data, } \\
\text { automatically actuate control signals to control a water pump or led lights, as well as sending the user notifications } \\
\text { when pertinent factors of the systems exceed or fall behind predetermined levels. }\end{array}$ \\
\hline
\end{tabular}


Additionally, the students were given opportunities to choose a cloud platform. In spring 2017 and spring 2018, the majority of students chose Amazon Webserver Services (AWS). There were other platforms used by the students such as Microsoft Azure, IBM (Watson), and Adafruit, etc. The students also were required to use Node-Red to design their dashboards. In respect to selecting a microcontroller, they used ESP8266, an inexpensive Arduino-based microcontroller with Wi-Fi capabilities.

Table 6. The list of topics covered in the IoT module

\begin{tabular}{|l|l|}
\hline Hour & Covered topic \\
\hline 1 & Introduction to IoT, concepts, challenges, applications \\
\hline 1 & Internet Principal, OSI model, IoT networking, IPv4/IPv6,TCP/UDP \\
\hline 1.5 & IoT communication protocol (ex: Wi-Fi, Bluetooth, 6LowPAN,Zigbee, Z-Wave, HART,etc) \\
\hline 1.5 & IoT transport layer protocols/messaging (ex: MQTT, CoAP, AMQP, XMPP,DDS) \\
\hline Total: 5 & \\
\hline
\end{tabular}

\section{IoT Resources}

An IoT system may contain a number of connected objects, which each has its own constraints such as radio range, data rate, power usage, etc. There are various communication protocols to address these restrictions. A review on the communication protocols for IoT devices is covered by the module using $[8,9]$. In addition to the communication protocol, messaging is another important topic that needed to be discussed in this module. Choosing the right messaging solution, architecture and the message/data sharing requirements are important topics that the students learn in the module. For example, AMQP and JMS have been designed to address applications requiring fast and reliable business transactions, whereas MQTT provides a simple and lightweight device data collection solutions. A good reference on the messaging technology can be found in [10]. There are also many references to cover IoT concepts, challenges, networking, and design principals for connected devices $[1,11,12]$.

\section{Conclusion}

The Internet of Things is a rapidly growing field and many companies are interested in hiring students with the IoT background. Many engineering schools have been integrating the IoT into their curricula. Some schools are offering the IoT as an independent undergraduate or a graduate course, whereas the rest have been developing IoT modules and have added them to one of the existing courses. The IoT content includes both hardware and software. An IoT module can be added to a hardware class such as embedded systems or M\&I course, or to be an auxiliary to a programming course. We developed an IoT module and have integrated into our M\&I course. In this class, students are required to develop a sensing device, and the IoT module helps them to add remote sensing/controlling to their products. The students have learned the material quickly and have shown significant interest in the IoT topics. 


\section{References}

[1] McEwen, Adrian, and Hakim Cassimally. Designing the internet of things. John Wiley \& Sons, 2013.

[2] Koo, Simon GM. "An integrated curriculum for Internet of Things: Experience and evaluation." Frontiers in Education Conference (FIE), 2015 IEEE. IEEE, 2015.

[3] Orser, David John, Bazargan, Kia, Sartori John. "Harnessing State-of-the-art Internet of Things Labs to Motivate First-year Electrical and Computer Engineering Students." Proceedings of the 2018 American Society of Engineering Education Annual Conference \& Exposition. 2018.

[4] Raikar, Meenaxi M., Padmashree Desai, and Jayalakshmi G. Naragund. "Active learning explored in Open elective course: Internet of Things (IoT)." Technology for Education (T4E), 2016 IEEE Eighth International Conference on. IEEE, 2016.

[5] Zhongmei, Ma, Sun Juan, and Li Qi. "Discussion on Curriculum and Practices of IoT Professional Course [J]." Microcontrollers \& Embedded Systems 10 (2011): 000.

[6] Morris, Alan S. "Measurement and instrumentation principles." (2001): 1743.

[7] Northrop, Robert B. Introduction to instrumentation and measurements. CRC press, 2005.

[8] Al-Sarawi, Shadi, et al. "Internet of Things (IoT) communication protocols." Information Technology (ICIT), 2017 8th International Conference on. IEEE, 2017.

[9] Salman, Tara, and Raj Jain. "Networking protocols and standards for internet of things." Internet of Things and Data Analytics Handbook (2015) (2015): 215-238.

[10] Foster, Andrew. "Messaging Technologies for the Industrial Internet and the Internet of Things." PrismTech Whitepaper(2015).

[11] Greengard, Samuel. The internet of things. MIT Press, 2015.

[12] Atzori, Luigi, Antonio Iera, and Giacomo Morabito. "The internet of things: A survey." Computer networks 54.15 (2010): 2787-2805. 